\title{
Flux variability analysis approach of autism related metabolism in stoichiometric model of mitochondria
}

\author{
Agris Pentjuss ${ }^{1,2}$, Oskars Rubenis $^{1 *}$, Daiga Bauze ${ }^{3}$, Lilija Aprupe ${ }^{4}$, Baiba Lace ${ }^{5}$ \\ ${ }^{1}$ Biosystems Group, Department of Computer Systems, Latvia University of Agriculture, \\ Liela iela 2, LV3001, Jelgava, Latvia \\ ${ }^{2}$ SIA TIBIT, Dobeles iela 10-9, LV3001, Jelgava, Latvia \\ ${ }^{3}$ Children's University Hospital, Child Psychiatry Clinic and Medical Genetics Clinics, Juglas iela 20, LV1079, Riga, Latvia \\ ${ }^{4}$ Faculty of Biosciences, University of Heidelberg, Neuenheimer Feld 234, D-69120, Heidelberg, Germany \\ ${ }^{5}$ Latvian Biomedical Research and Study Center, Ratsupites iela 1, LV1067, Riga, Latvia
}

*Corresponding author

oskars.rubenis@gmail.com

Received: 20 November 2013; accepted: 28 November 2013; published online: 29 November 2013.

This paper has supplementary material.

\begin{abstract}
Autism spectrum disorders are early childhood neurodevelopmental disorders, it is not a disease but rather a syndrome that is characterized by a multifactorial type of inheritance and a rapid annual increase in prevalence. In some cases autism spectrum disorders are one of the symptoms of monogenic or chromosomal pathology, and can also be a symptom of inherited metabolic disorders.

A reconstruction of human mitochondrial metabolic network has been used to model insufficiency of some genes encoded proteins to observe the consequences at metabolic network scale and propose approaches for the development of diagnostics. Flux variability analysis of mitochondrial metabolic network reconstruction is performed maximizing ATP production. It is found that deletion of SUCLG2 gene reduces the maximal production of ATP by 50\% with wide flux variability range for most of reactions. Deletion of gene SLC25A12 reduces the maximal ATP production just by $1 \%$ but the new state is very fragile as most of reactions have very narrow flux variability range and any disturbance would cause reduction of ATP production that can not be compensated by other reactions. Detection of insufficiency of genes SUCLG2 and SLC25A12 is suggested by observing of fluxes of specific reactions found by flux variability analysis.

The reactions with non-overlapping flux variability range can be very informative for the diagnostics of deficiency of particular gene. Still the experiments demonstrated that there are just few reactions with non-overlapping range. The analysis of wide flux variability range in case of deletion also can be perspective as detection of zero flux could be easier task for experimental detection. Pathway scale analysis could bring new constraints for improvement of the model and increase the number of reactions with non-overlapping flux variability range.
\end{abstract}

Keywords: Autism spectrum disorders, constraint based reconstruction, mitochondrion, flux variability analysis, modeling.

\section{Introduction}

Autism spectrum disorders (ASD) are serious early childhood neurodevelopmental disorders with unknown etiology and a rapid annual increase in prevalence. ASD are a broad phenotype including less severe disorders (Johnson and Myers, 2007). ASD are clinically characterized by impaired social and communication skills, hyperactivity and attention deficit, stereotypic movements and interests, stereotypic rituals, emotional disturbances, and varying degrees of expressive and receptive language development disorders (Risch et al., 1999).

ASD is not a disease but rather a syndrome that is characterized by a multifactorial type of inheritance. In some cases, it is one of the symptoms of monogenic or chromosomal pathology, and can also be a symptom of inherited metabolic disorders. In early childhood, the latter can manifest primarily with autistic behavior, speech development delay or regression, and mental retardation. This collection of symptoms should be classified as ASD (Gillberg, 2006).

One of the first hypothesis about mitochondrial dysfunction associated with ASD was published in 1998 by Lombard
(Lombard, 1998). Mitochondria are cell structures, which have role in the several functions in cell: ATP synthesis, fatty acid oxidation, heme biosynthesis, heat generation, calcium homeostasis, citric acid cycle and apoptosis (Koopman et al., 2012). Hypothesis was based on the frequently observed lactic acidosis and carnitine deficiency in patients with ASD. With a rough study based on the elevation of the lactic acid and morphological criteria on the muscle biopsy in the ASD patients, researchers established prevalence of mitochondrial dysfunction in ASD group. That was 7.2\% (Oliveira et al., 2005). In the clinical review of the patients with mitochondrial dysfunction it was concluded, that in 25 of them primary diagnosis was ASD. The most common defect was decreased activity of complex I (Weissman et al., 2008).

Rossignol et al. in the paper of the meta-analysis concluded that mitochondrial dysfunction is the most common metabolic abnormality associated with ASD. It was estimated to be 5\%, when compared to the general population of $0.01 \%$. Most of the identified cases were not associated with genetic abnormalities in mtDNA, but resulted most probably of the secondary 
mitochondrial dysfunction (Rossignol and Frye, 2012). Postmortem brain biopsies in ASD patients showed decreased Complex I and Complex IV activities (Tang et al., 2013).

Significant input in the ASD research comes from the genome wide analysis, which helped to reveal or confirm some strong ASD candidate genes also involved in the mitochondrial metabolism. Gene SUCLG2 was described as significantly associated with ASD from the genome wide analysis of the exonic copy number variants (Bucan et al., 2009). Two single nucleotide polymorphisms (SNPs) that showed evidence for divergent distribution between autistic and nonautistic subjects were identified, both within $S L C 25 A 12$, a gene encoding the mitochondrial aspartate/glutamate carrier $(A G C 1)$. In the second stage, the two SNPs in SLC25A12 were further genotyped in 411 autistic families, and linkage and association tests were carried out in the 197 informative families. A strong association of autism with SNPs within the SLC25A12 gene was demonstrated (Ramoz et al., 2004). However conflicting results were obtained in the group of ASD patients from Protugal. They found no evidence of SLC25A12 gene involvement is responsible of hyperlacticidemia reported previously (Correia et al., 2006).

The above mentioned studies indicate necessity to discover the mechanisms of ASD at metabolic level. Modeling can be one of tools for the studies of complicated mechanisms applying different modeling approaches (Stelling, 2004). Reconstructions of metabolic networks represent stoichiometrically balanced reactions that are possible in particular organism based on genome annotations, literature, experimental and other data. Reconstructions do not include data about the kinetics of reactions and concentrations of metabolites. Addition of numerical information about particular environment and organism state characteristic upper and lower limits of reaction fluxes transforms the reconstruction into a state and environment specific model. The behavior of model then can be compared to experimental data. More than 50 genome-scale reconstructions and models of metabolism of different organisms have been developed in last decades (Oberhardt et al., 2009). Protocols for their development of reconstructons have been published as well (Thiele and Palsson, 2010). Reconstructions for a number of human tissues have been developed (Agren et al., 2012). The recent achievement in reconstruction building is the Recon2 (Thiele et al., 2013) which can be used for studies of human physiology and pathology (Bordbar and Palsson, 2012).

Also human mitochondrion has been reconstructed and modeled. The most comprehensive mitochondria devoted reconstruction so far has been published (Vo et al., 2004) improved and analyzed afterwards (Thiele et al., 2005; Vo, 2007). There are also available dynamic models of mitochondria with limited scope of reactions (Bertram et al., 2006; Wu et al., 2007).

To make in silico experiments with reconstruction based human mitochondrial model (Thiele et al., 2005) we used $C O$ BRA Toolbox v2.0 (Schellenberger et al., 2011) for Matlab. COBRA is used for analysis of metabolic models, signaling models and even genome scale reconstructions of different organisms. The models contain necessary metabolites and reactions, for the specific organism. The necessary biological and environmental constraints can be found in different scientific literature, databases or by laboratory experiments.
The analysis of models and reconstructions are based on different methods like Flux Balance Analysis (Orth et al., 2010), Flux Variability Analysis (Burgard et al., 2001; Kostromins, 2012). With Flux Balance Analysis (FBA) it is possible to find out the best possible flux solution in the model for particular objective function, for example fat synthesis in adipose tissue from glucose (Fell and Small, 1986). Although this method shows only one of the many best solutions, it is very fast calculation method and takes only few seconds to get the results (Orth et al., 2010). Flux Variability Analysis (FVA) shows minimum and maximum flux of reactions in the network to maintain some state of the network (Gudmundsson and Thiele, 2010). It is quite informative method when there is need to find out not exact state of metabolism, but complete solution space has to be scanned for analysis purposes. AltFluxes toolbox for COBRA (Kostromins, 2012) performs Flux Variability Analysis with a user friendly interface, and results are visualized and easier interpretable. Combination of different methods gives additional opportunities (Lewis et al., 2012). The described approaches can be used to analyze the consequences of different changes in the network, including gene insufficiency or deletion.

In this study the impact of insufficiency of two possibly autism related genes $S L C 25 A 12$ (Correia et al., 2006; Ramoz et al., 2004) and SUCLG2 (Bucan et al., 2009) are examined by methods of stoichiometric analysis to find out the metabolically traceable side effects of deficiency in genes encoded proteins that could be easily observable thus enabling diagnostics and determination of cause-effect relationships. Analysis is performed mainly using COBRA Toolbox2 (Schellenberger et al., 2011) for Matlab. The diagnostics of insufficiency of genes SLC25A12 and SUCLG2 is suggested by analyzing parts of metabolic pathways found by constraint based modeling. It is found that deletion of gene $S L C 25 A 12$ reduces the maximal $A T P$ production just by $1 \%$ while deletion of $S U C L G 2$ in the model reduces the maximal production of $A T P$ by $50 \%$.

\section{Materials and methods}

In this study the human mitochondrial metabolic network reconstruction developed in Palsson Lab is used (Thiele et al., 2005). This reconstruction is extension of earlier work of the same group (Vo et al., 2004). Reconstruction of mitochondrion in our study consists of 225 biochemical reactions and 235 metabolites (121 mitochondrial, 89 cystolic, and 25 extracellular). Involved metabolites and their coding genes were crossreferenced with scientific publications about autism and autism spectrum disorders and publicly available databases (AutDb, (http://autism.mindspec.org/autdb/Welcome.do) Ndar (http://ndar.nih.gov). In all calculations $\mu \mathrm{mol} / \mathrm{min} / \mathrm{g}$ of proteins is used as flux measurement unit as it was done in the initial model (Thiele et al., 2005). Two genes SLC25A12 (Correia et al., 2006; Ramoz et al., 2004) and SUCLG2 (Bucan et al., 2009) were associated with ASD and found in the reconstructed metabolic network, thus exclusion criteria was not developed. The names of reactions are associated with reactions and metabolites as in the supplementary material 1 based on the earlier published reconstruction (Thiele et al., 2005). The supplementary material 1 is an excel file in COBRA readable format and can be opened using function xls2model.

Gene SLC25A12 (solute carrier family 25 (aspartate/glutamate carrier), member 12) is localized on chromosome two long arm locus 24 , and its coded protein is involved 
in the exchange of $\mathrm{Ca}(2+)$-bound aspartate for glutamate across the inner mitochondrial membrane. Knock-out mice for the gene SLC25A12 model showed global cerebral hypomyielination (Jalil et al., 2005).

Gene SUCLG2 (succinate-CoA ligase, GDP-forming, beta subunit) spans on the chromosome three short arm region 14.1 and it encodes beta subunit of succinyl-CoA synthethase, which catalyzes a reversible reaction involving energy production through ATP or GTP. Phenotype data for the haploinsufficiency of the gene is not available, however alpha subunit deficiency can cause severe lactic acidemia (Ostergaard et al., 2007).

The studied genes are encoding enzymes of reactions represented in the reconstruction. The gene SLC25A12 corresponds to the reaction $A S P G L U m$ and gene SUCLG2 corresponds to the reaction $S U C C O A S m$.

Flux variabaility analysis (Gudmundsson and Thiele, 2010) is performed using COBRA 2 Toolbox for Matlab (Schellenberger et al., 2011) and AltFluxes toolbox (Kostromins, 2012) for COBRA to calculate and visualize the flux variability range. The flux variability analysis is calculated for a particular state of a network. In this study it is calculated for maximal ATP production rate (reaction DM_atp $(c)$ ). Upper and lower bounds of all steady state reaction fluxes can be determined using flux variability, where setting objective function flux for maximal value, each reaction flux is minimized and maximized to find steady state bounds.

Hypergraph layouts for visualization of gene deletion effects on steady state flux distributions are made using Paint4Net toolbox for COBRA (Kostromins and Stalidzans, 2012).

\section{Results and discusion}

Three cases of ATP production (reaction DM_atp(c)) maximization are investigated in this research by flux variability analysis: 1) unaffected model (Thiele et al., 2005), 2) deletion of reaction ASPGLUm corresponding gene SLC25A12 and 3) deletion of reaction $S U C C O A S m$ corresponding gene $S U$ $C L G 2$. The deletion is implemented as setting the upper and lower bounds of reactions to zero for particular reaction.

Flux variability analysis (FVA) is performed with AltFluxes toolbox (Kostromins, 2012). The results are summarized in different sheets of supplementary material 2. The FVA results of all reactions are summarized in sheet "All_data".

Some reactions are considered as not informative because of no changes or small changes of flux variability range between three simulated cases and therefore are excluded from further analysis. 60 reactions with flux variability range changes between unaffected and two gene deletion cases experiments more than 5 units are selected for further analysis. Their values for all three cases are summarized in Fig. 1 and in the sheet "Analysis" of Supplementary materials 2.

\subsection{Comparison of deletion of reaction ASPGLUm corresponding gene SLC25A12 with unaffected case model}

The deletion of $S L C 25 A 12$ gene causes small reduction in the network ability to maximize ATP production compared to unaffected case model. The maximal ATP production drops from 68.4 to 67.7 . The relative change is just about $1 \%$ which is hard to recognize phenotypically or by measurements.
At the same time the variability of fluxes changes significantly (Fig. 1, unaffected case and ASPGLUm case). Relatively wide flux variability ranges of the unaffected case indicate flexibility and robustness (Vo, 2007) of the network to produce 68.4 units of ATP. The changes in flux range of one reaction can be compensated by adaptation of other reactions. In the case of deleted ASPGLUm the flux variability of most of reactions is very small, represented by a line (Fig. 1). That means any limitations in any of those reactions will cause reduction of ATP production. In other words there is very limited number of alternatives and the ATP production has stayed almost at the same level as in unaffected case but has become very fragile. In case of unaffected model there are just two nonzero fluxes without significant variability: CYOR-u1Om and SUCD3$u 10 \mathrm{~m}$. In case of $A S P G L U m$ the number of such reactions increase to 50 out of 60 indicating loss of flexibility and robustness.

\subsection{Comparison of deletion of reaction SUCCOASm corresponding gene SUCLG2 with unaffected case model}

The deletion of SUCLG2 gene causes about $50 \%$ reduction in the network ability to produce ATP. The maximal ATP production drops from 68.4 to 34.0. At the same time the large reduction of maximal production capacity has brought also large spectrum of alternatives and 34.0 units of ATP can be produced in many ways using alternative paths demonstrating flexibility and robustness. The flux variability ranges are higher that in the unaffected case. Only one nonzero reaction PIt $2 \mathrm{~m}$ has to perform at particular speed to keep 34.0 units of ATP production. In addition many reactions with nonzero values in unaffected case and ASPGLUm deletion case can have even zero values in case of reaction SUCCOASm deletion. There are just a couple of reactions that have to be at nonzero fluxes (ASPTAm and CSm). That means deletion of any other reaction out of 60 reactions of interest would not cause further reduction of maximal ATP production below 34.0 units.

Another feature of deletion of reaction SUCCOASm corresponding gene $S U C L G 2$ is the fact that flux variability range of reaction PIt $2 m$ has no overlap with other two cases. After deletion the range is 32-33 units while in case of unaffected model it is 48-99 and in ASPGLUm case it is 50-70. This nonoverlapping flux variability range could be used for diagnostics purposes. In our model reaction $S U C C O A S m$ utilizes inorganic phosphate in the reversible reaction with the formation of nucleoside triphosphate (NTP) from nucleoside diphosphate (NDP) and inorganic phosphate. We can speculate that partial failure of reaction PIt $2 \mathrm{~m}$ in the mitochondrial metabolic network model is caused by inorganic phosphate deficiency.

Also carbon dioxide transport from mitochondria to citoplasm in unaffected case (the flux bounds 100-132) and SUCCOASm reaction deletion case (the flux bounds 7 - 128) shows steady state possibilities with even 10 times smaller flux. This allows to speculate that decreased flux in mitochondiral carbon dioxide transport shows decreased TCA cycle overall flux in SUCCOASm deletion case, because most of all carbon dioxide in mitochondria is produced in TCA cycle. 


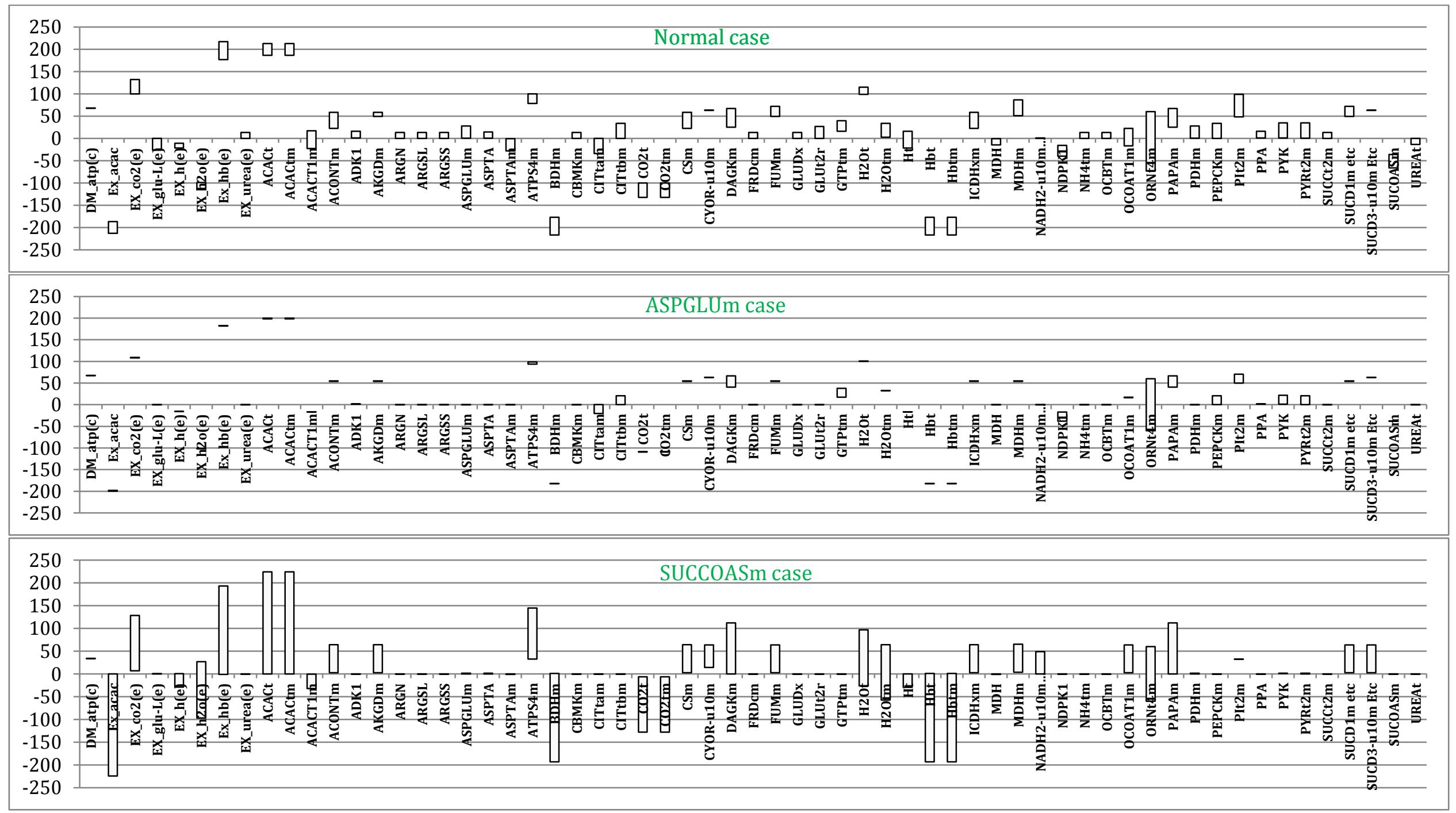

Fig. 1. The results of flux variability analysis for three cases maximizing ATP production: 1) unaffected mitochondria model, 2) deletion of SUCLG2 and 3) deletion of $S L C 25 A 12$. The units of fluxes are $\mu \mathrm{mol} / \mathrm{min} / \mathrm{g}$ of proteins. 


\subsection{Advantages and limitations of approach}

The presented analysis of gene deletions has been based on the flux variability analysis assuming that the network aims to maximize ATP production. More complex approach with several criteria should be used to assess the multifunctionality of the network. Production of heme, phospholipids (Vo et al., 2004) and others should be taken into account as well. That could be done fixing reaction rates for some branches to ensure production of some metabolites. This kind of analysis performed for several other objective functions that are critical for the functionality of mitochondria could give additional results or could indicate need of improvement of the model.

Another drawback of the proposed approach is the fact that the reduction of ATP production capacity in case of suboptimal fluxes (not all reactions can have fluxes within flux variability range) may be large or small. In case of small reduction the deletion impact would be less observable than in case of sharp decrease of ATP production. On the other hand even more promising would be analysis of the newest measurements of mitochondrial metabolism giving additional constraints to the model thus improving it's quality.

The reactions with non-overlapping flux variability range can be very informative for the diagnostics of deficiency of particular gene. Still the experiments demonstrated that there are just few reactions with non-overlapping range. Even in those cases the difference between the flux ranges of reactions may be hard to measure the difference with necessary accuracy.

The analysis of widening of the flux variability range also can be perspective as detection of zero flux could be easier task experimentally. Adding on that the layer of pathway scale analysis it could be possible to develop new constraints for improvement of the model that could lead to increase of reactions with non-overlapping flux variability range.

The above described approach of flux variability analysis could be efficient for other processes of interest depending on the accuracy of model and peculiarities of he process. The advantages of the approach are the small amount of necessary calculations because of application of linear algebra methods and possibility to analyze medium scale and even larger models.

\section{Conclusion}

Flux variability analysis of reconstruction based model of mitochondrial metabolism and model with deleted SLC25A12 gene show very similar results. The deletion of $S L C 25 A 12$ causes the reductions of maximal ATP production from 68.4 to 67.7 that is about one percent and would be hardly observable. At the same time the deletion reduces the flexibility and robustness of network as capacity limitations of any reaction can lead to decrease of maximal ATP production.

The deletion of $S U C L G 2$ gives decrease of ATP production from 68.4 to 34.0. The Flux variability analysis shows mostly wider range of values for the model with deletion. There are some branches which may have no or very small flux. That is a way how the malfunction of SUCLG2 could be detected. One reaction has not overlapping flux variability range that can be used for diagnostic purposes.

Analysis of stoichiometric models in some cases can give useful diagnostic hints to find out the level of influence of in- sufficiency of particular genes by determining gene deletion specific profile of metabolism.

That can be done by determination of reactions with nonoverlapping flux variability range comparing performance of unaffected model and model with gene deletion or analyzing the widening of flux variability range towards zero fluxes

\section{References}

Agren, R., Bordel, S., Mardinoglu, A., Pornputtapong, N., Nookaew, I. and Nielsen, J. (2012), "Reconstruction of genome-scale active metabolic networks for 69 human cell types and 16 cancer types using INIT" PLoS computational biology, Vol. 8 No. 5, p. e1002518. http://dx.doi.org/10.1371/journal.pcbi.1002518

Bertram, R., Gram Pedersen, M., Luciani, D.S. and Sherman, A. (2006), "A simplified model for mitochondrial ATP production" Journal of theoretical biology, Vol. 243 No. 4, pp. 575-86. http://dx.doi.org/10.1016/j.jtbi.2006.07.019

Bordbar, A. and Palsson, B.O. (2012), "Using the reconstructed genome-scale human metabolic network to study physiology and pathology" Journal of internal medicine, Vol. 271 No. 2, pp. 131-41. http://dx.doi.org/10.1111/j.1365-2796.2011.02494.x

Bucan, M., Abrahams, B.S., Wang, K., Glessner, J.T., Herman, E.I., Sonnenblick, L.I., Alvarez Retuerto, A.I., et al. (2009), "Genome-wide analyses of exonic copy number variants in a family-based study point to novel autism susceptibility genes" PLoS genetics, Vol. 5 No. 6, p e1000536. http://dx.doi.org/10.1371/journal.pgen.1000536

Burgard, a P., Vaidyaraman, S. and Maranas, C.D. (2001), "Minimal reaction sets for Escherichia coli metabolism under different growth requirements and uptake environments" Biotechnology progress, Vol. 17 No. 5, pp. 791-7. http://dx.doi.org/10.1021/bp0100880

Correia, C., Coutinho, A.M., Diogo, L., Grazina, M., Marques, C., Miguel, T., Ataíde, A., et al. (2006), "Brief report: High frequency of biochemical markers for mitochondrial dysfunction in autism: no association with the mitochondrial aspartate/glutamate carrier SLC25A12 gene" Journal of autism and developmental disorders, Vol. 36 No. 8, pp. 1137-40. http://dx.doi.org/10.1007/s10803-006-0138-6

Fell, D. and Small, J. (1986), "Fat synthesis in adipose tissue. An examination of stoichiometric constraints" Biochemical journal, Vol. 238, pp. 781786.

Gillberg, C. (2006), "Autism spectrum disorders", in Gillberg, C., Harrington, R. and Steinhausen, H. (Eds.), A Clinician's Handbook of Child and Adolescent Psychiatry, Cambridge University Press, Cambridge UK; New York, pp. 447-489. http://dx.doi.org/10.1017/CBO9780511543807.017

Gudmundsson, S. and Thiele, I. (2010), "Computationally efficient flux variability analysis" BMC bioinformatics, Vol. 11 No. 1, p. 489 http://dx.doi.org/10.1186/1471-2105-11-489

Jalil, M.A., Begum, L., Contreras, L., Pardo, B., Iijima, M., Li, M.X., Ramos, M., et al. (2005), "Reduced N-acetylaspartate levels in mice lacking aralar, a brain- and muscle-type mitochondrial aspartate-glutamate carrier" The Journal of biological chemistry, Vol. 280 No. 35, pp. 31333-9. http://dx.doi.org/10.1074/jbc.M505286200

Johnson, C.P. and Myers, S.M. (2007), "Identification and evaluation of children with autism spectrum disorders" Pediatrics, Vol. 120 No. 5, pp. 1183-215. http://dx.doi.org/10.1542/peds.2007-2361

Koopman, W.J.H., Willems, P.H.G.M. and Smeitink, J.A.M. (2012), "Monogenic mitochondrial disorders" The New England journal of medicine, Vol. $366 \quad$ No. 12, pp. 1132-41. http://dx.doi.org/10.1056/NEJMra1012478

Kostromins, A. (2012), "Altfluxes: COBRA Toolbox extension for flux variability analysis of stoichiometric models of metabolism" 5th International Scientific Conference on Applied Information and Communication Technologies, pp. 294-299.

Kostromins, A. and Stalidzans, E. (2012), "Paint4Net: COBRA Toolbox extension for visualization of stoichiometric models of metabolism" Biosystems, Vol. $109 \quad$ No. 2, pp. 233-9. http://dx.doi.org/10.1016/j.biosystems.2012.03.002

Lewis, N.E., Nagarajan, H. and Palsson, B.O. (2012), "Constraining the metabolic genotype-phenotype relationship using a phylogeny of in silico methods" Nature reviews. Microbiology, Nature Publishing Group, Vol. 10 No. 4, pp. 291-305.

Lombard, J. (1998), “Autism: a mitochondrial disorder?" Medical hypotheses, Vol. 50 No. 6, pp. 497-500. http://dx.doi.org/10.1016/S03069877(98)90270-5 
Oberhardt, M. a, Palsson, B.O. and Papin, J. a. (2009), "Applications of genome-scale metabolic reconstructions" Molecular systems biology, Vol. 5 No. 320 , p. 320.

Oliveira, G., Diogo, L., Grazina, M., Garcia, P., Ataíde, a, Marques, C., Miguel, T., et al. (2005), "Mitochondrial dysfunction in autism spectrum disorders: a population-based study" Developmental medicine and child neurology, Vol. $47 \quad$ No. $\quad 3, \quad$ pp. $185-9$. http://dx.doi.org/10.1017/S0012162205000332

Orth, J.D., Thiele, I. and Palsson, B.O. (2010), "What is flux balance analysis?" Nature biotechnology, Vol. 28 No. 3, pp. 245-8. http://dx.doi.org/10.1038/nbt.1614

Ostergaard, E., Christensen, E., Kristensen, E., Mogensen, B., Duno, M., Shoubridge, E. a and Wibrand, F. (2007), "Deficiency of the alpha subunit of succinate-coenzyme A ligase causes fatal infantile lactic acidosis with mitochondrial DNA depletion" American journal of human genetics, Vol. 81 No. 2, pp. 383-7. http://dx.doi.org/10.1086/519222

Ramoz, N., Reichert, J.G., Smith, C.J., Silverman, J.M., Bespalova, I.N., Davis, K.L. and Buxbaum, J.D. (2004), "Linkage and association of the mitochondrial aspartate/glutamate carrier SLC25A12 gene with autism" The American journal of psychiatry, Vol. 161 No. 4, pp. 662-9. http://dx.doi.org/10.1176/appi.ajp.161.4.662

Risch, N., Spiker, D., Lotspeich, L., Nouri, N., Hinds, D., Hallmayer, J., Kalaydjieva, L., et al. (1999), "A genomic screen of autism: evidence for a multilocus etiology" American journal of human genetics, Vol. 65 No. 2, pp. 493-507. http://dx.doi.org/10.1086/302497

Rossignol, D. a and Frye, R.E. (2012), "Mitochondrial dysfunction in autism spectrum disorders: a systematic review and meta-analysis" Molecular psychiatry, Nature Publishing Group, Vol. 17 No. 3, pp. 290-314. http://dx.doi.org/10.1038/mp.2010.136

Schellenberger, J., Que, R., Fleming, R.M.T., Thiele, I., Orth, J.D., Feist, A.M., Zielinski, D.C., et al. (2011), "Quantitative prediction of cellular metabolism with constraint-based models: the COBRA Toolbox v2.0", Nature Protocols, Vol. 6 No. 9, pp. 1290-1307. http://dx.doi.org/10.1038/nprot.2011.308
Stelling, J. (2004), "Mathematical models in microbial systems biology" Current opinion in microbiology, Vol. 7 No. 5, pp. 513-8. http://dx.doi.org/10.1016/j.mib.2004.08.004

Tang, G., Gutierrez Rios, P., Kuo, S.-H., Akman, H.O., Rosoklija, G., Tanji, K., Dwork, A., et al. (2013), "Mitochondrial abnormalities in temporal lobe of autistic brain" Neurobiology of disease, Elsevier Inc., Vol. 54, pp. $349-61$.

Thiele, I. and Palsson, B.O. (2010), "A protocol for generating a high-quality genome-scale metabolic reconstruction" Nature protocols, Vol. 5 No. 1, pp. 93-121. http://dx.doi.org/10.1038/nprot.2009.203

Thiele, I., Price, N.D., Vo, T.D. and Palsson, B.O. (2005), "Candidate metabolic network states in human mitochondria. Impact of diabetes, ischemia, and diet" The Journal of biological chemistry, Vol. 280 No. 12, pp. 11683-95. http://dx.doi.org/10.1074/jbc.M409072200

Thiele, I., Swainston, N., Fleming, R.M.T., Hoppe, A., Sahoo, S., Aurich, M.K., Haraldsdottir, H., et al. (2013), "A community-driven global reconstruction of human metabolism" Nature Biotechnology, No. September 2012, doi:10.1038/nbt.2488. http://dx.doi.org/10.1038/nbt.2488

Vo, T.D. (2007), Systems analysis of energy metabolism elucidates the roles of mitochondria in human health and disease, University of California.

Vo, T.D., Greenberg, H.J. and Palsson, B.O. (2004), "Reconstruction and functional characterization of the human mitochondrial metabolic network based on proteomic and biochemical data" The Journal of biological chemistry, Vol. 279 No. 38, pp. 39532-40. http://dx.doi.org/10.1074/jbc.M403782200

Weissman, J.R., Kelley, R.I., Bauman, M.L., Cohen, B.H., Murray, K.F. Mitchell, R.L., Kern, R.L., et al. (2008), "Mitochondrial disease in autism spectrum disorder patients: a cohort analysis" PloS one, Vol. 3 No. 11, p. e3815. http://dx.doi.org/10.1371/journal.pone.0003815

Wu, F., Yang, F., Vinnakota, K.C. and Beard, D. a. (2007), "Computer modeling of mitochondrial tricarboxylic acid cycle, oxidative phosphorylation, metabolite transport, and electrophysiology" The Journal of biological chemistry, Vol. 282 No. 34, pp. 24525-37. http://dx.doi.org/10.1074/jbc.M701024200 